\title{
Therapeutic drug monitoring-guided continuous infusion of piperacillin/tazobactam significantly improves pharmacokinetic target attainment in critically ill patients: a retrospective analysis of four years of clinical experience
}

\author{
Daniel C. Richter ${ }^{1} \mathbb{C} \cdot$ Otto Frey $^{2}$. Anka Röhr ${ }^{2}$. Jason A. Roberts ${ }^{3,4,5,6}$. Andreas Köberer ${ }^{7}$. Thomas Fuchs ${ }^{7}$. \\ Nikolaos Papadimas ${ }^{8} \cdot$ Monika Heinzel-Gutenbrunner $^{9} \cdot$ Thorsten Brenner $^{1} \cdot$ Christoph Lichtenstern $^{1}$. \\ Markus A. Weigand ${ }^{1} \cdot$ Alexander Brinkmann $^{7}$
}

Received: 25 June 2019 / Accepted: 22 August 2019 / Published online: 31 August 2019

(C) The Author(s) 2019

\begin{abstract}
Purpose Standard dosing and intermittent bolus application (IB) are important risk factors for pharmacokinetic (PK) target non-attainment during empirical treatment with $\beta$-lactams in critically ill patients, particularly in those with sepsis and septic shock. We assessed the effect of therapeutic drug monitoring-guided (TDM), continuous infusion (CI) and individual dosing of piperacillin/tazobactam (PIP) on PK-target attainment in critically ill patients.

Methods This is a retrospective, single-center analysis of a database including 484 patients [933 serum concentrations (SC)] with severe infections, sepsis and septic shock who received TDM-guided CI of PIP in the intensive care unit (ICU) of an academic teaching hospital. The PK-target was defined as a PIP SC between 33 and $64 \mathrm{mg} / \mathrm{L}$ [ $f \mathrm{~T}>2-4$ times the epidemiological cutoff value (ECOFF) of Pseudomonas aeruginosa (PSA)].

Results PK-target attainment with standard dosing (initial dose) was observed in 166 patients (34.3\%), whereas only 49 patients $(10.1 \%)$ demonstrated target non-attainment. The minimum PK-target of $\geq 33 \mathrm{mg} / \mathrm{L}$ was overall realized in $89.9 \%$ $(n=435 / 484)$ of patients after the first PIP dose including 146 patients $(30.2 \%)$ with potentially harmful SCs $\geq 100 \mathrm{mg} / \mathrm{L}$. Subsequent TDM-guided dose adjustments significantly enhanced PK-target attainment to 280 patients (62.4\%) and significantly reduced the fraction of potentially overdosed $(\geq 100 \mathrm{mg} / \mathrm{L})$ patients to $4.5 \%(n=20 / 449)$. Renal replacement therapy (RRT) resulted in a relevant reduction of PIP clearance $\left(\mathrm{CL}_{\mathrm{PIP}}\right)$ : no RRT CL $\mathrm{PIP}_{\mathrm{P}} 6.8 / 6.3 \mathrm{~L} / \mathrm{h}$ (median/IQR) [SCs $n=752$, patients $n=405$ ], continuous veno-venous hemodialysis (CVVHD) $\mathrm{CL}_{\mathrm{PIP}} 4.3 / 2.6 \mathrm{~L} / \mathrm{h}$ [SCs $n=160, n=71$ patients], intermittent hemodialysis (iHD) $\mathrm{CL}_{\mathrm{PIP}} 2.6 / 2.3 \mathrm{~L} / \mathrm{h}$ [SCs $n=21, n=8$ patients]). A body mass index (BMI) of $>40 \mathrm{~kg} / \mathrm{m}^{2}$ significantly increased $\mathrm{CL}_{\mathrm{PIP}} 9.6 / 7.7 \mathrm{~L} / \mathrm{h}$ [SC $n=43, n=18$ patients] in these patients. Age was significantly associated with supratherapeutic PIP concentrations $(p<0.0005)$, whereas high CrCL led to non-target attainment $(p<0.0005)$. Patients with target attainment (33-64 mg/L) within the first $24 \mathrm{~h}$ exhibited the lowest hospital mortality rates $(13.9 \%[n=23 / 166]$, $p<0.005)$. Those with target non-attainment demonstrated higher mortality rates $(\leq 32 \mathrm{mg} / \mathrm{L} ; 20.8 \%[n=10 / 49] \geq 64 \mathrm{mg} / \mathrm{L}$; $29.4 \%[n=79 / 269])$.

Conclusion TDM-guided CI of PIP is safe in critically ill patients and improves PK-target attainment. Exposure to defined PK-targets impacts patient mortality while lower and higher than intended SCs may influence the outcome of critically ill patients. Renal function and renal replacement therapy are main determinants of PK-target attainment. These results are only valid for CI of PIP and not for prolonged or intermittent bolus administration of PIP.
\end{abstract}

Keywords Continuous infusion $\cdot \beta$-lactams $\cdot$ PK/PD $\cdot$ Customized drug dosing $\cdot$ Critical illness $\cdot$ Sepsis

Daniel C. Richter

daniel.richter@med.uni-heidelberg.de

$\triangle$ Alexander Brinkmann

alexander.brinkmann@kliniken-heidenheim.de

Extended author information available on the last page of the article 


\section{Introduction}

\section{Background}

Early effective antibiotic treatment is a fundamental mainstay in sepsis therapy and should be initiated as soon as possible $[1,2]$ to reduce patient mortality [2-4]. $\beta$-lactams like PIP are frequently used antibiotics for empiric treatment of severe infections in the ICU [5]. Attaining defined pharmacokinetic targets within the first 24-48 h of treatment repeatedly crystallized as an important predictor of treatment success in sepsis [6-8]. Consistently, intermittent bolus applications (IB) of standard doses are recognized as a main contributor for target non-attainment and potentially unfavorable patient outcome [9, 10]. Given their hydrophilic properties, $\beta$-lactams and PIP especially are prone to rapid changes in renal function and volume of distribution both of which frequently occur in patients with sepsis and septic shock [11]. Zander et al. [12] found only recently infrequent target attainment during IB application of PIP, especially in patients with a high CrCL. CI infusion represents a reasonable approach of customized drug dosing in the ICU as to extend the time that the unbound fraction of PIP remains above the pathogen-specific minimum inhibitory concentration (MIC) $\left(f \mathrm{~T}_{>\mathrm{MIC}}\right)[6,7,13,14]$ up to $100 \%$ of the dosing interval $[10,12,15]$. Several studies demonstrated positive effects (clinical cure and survival rates) of CI compared to IB in the context of sepsis [16-22]. As evidence increases, the demand for dose optimization and TDM-guided individual dosing strategies grows [12, 23, 24]

\section{Objectives}

We analyzed a PK database of 484 patients with overall 933 SCs covering a period of 4 years of CI of $\beta$-lactams in an ICU. We primarily assessed PIP target attainment within the first $24 \mathrm{~h}$ of treatment and further investigated the effect of subsequent TDM-guided dose adjustments on PK-target attainment to support existing data regarding the safety of CI of $\beta$-lactams. Secondly, the influence of renal function, renal replacement therapy and obesity on PIP-PK was analyzed. Moreover, ICU- and hospital mortality data were evaluated in relation to PIP SCs.

\section{Methods}

\section{Study setting and population}

We retrospectively analyzed TDM-data of 484 patients ( $\geq 16$ years) admitted to an interdisciplinary German ICU of an academic teaching hospital between December 2008 and October 2012. Patients were admitted for treatment of severe infections, (severe) sepsis or septic shock. RRT was not an exclusion criterion. The study was approved by the ethics commission of the University of Ulm, Germany (Proposal number $351 / 14 ; 12 / 2014)$.

\section{Sampling and bioanalysis}

Patients received TDM-guided CI of PIP according to the following standardized protocol that derived from years of clinical experience and the routine use of PK simulations. An initial piperacillin/tazobactam bolus [2.25 g fixed (according to PK simulation data), 30-min infusion] was followed by immediate CI of piperacillin/tazobactam. The initial CI doses were determined according to the patients' renal function. The total amount of PIP within the first $24 \mathrm{~h}$ was $12 \mathrm{~g}$ [4 g;2-16 g] for all patients and never exceeded $16 \mathrm{~g}$ within the first $24 \mathrm{~h}$ of treatment. In the presence of septic shock, PIP was supplemented with ciprofloxacin. Initial TDM (referred to as day1) was performed under steady-state conditions ( $>4$ halftimes; minimum $>12 \mathrm{~h}$ after treatment initiation). PIP doses were subsequently adapted according to the results of PIP TDMs with PIP SCs of 33-64 mg/L being the primary PK-target $\left(f \mathrm{~T}_{>2-4 \text { ECOFF PSA }}\right)$. TDM-guided dose adjustments and consecutive TDMs were advised and supervised by clinical pharmacists. Adjustment to microbiological data and resistance testing was performed as soon as information was made available. PIP concentrations were measured using high-performance liquid chromatography (HPLC), as published before [25]. TDM-data were available and reported 2-4 $\mathrm{h}$ after the blood sample arrived in the laboratory (blood sample: 6-8 a.m., TDM-data reporting: 10-12 a.m.). The interpretation as well as dose adjustment recommendations were supervised by trained clinical pharmacists.

\section{Definitions}

Although using the combination of piperacillin and tazobactam (PIP/TAZ), HPLC detection was restricted to the total PIP fraction which is why we use the abbreviation PIP in the present manuscript. The difference between measured (total) and unbound fractions seems of interest only in the highly protein bound $\beta$-lactams [26]. The ECOFF of PIP for PSA is $16 \mathrm{mg} / \mathrm{L}$. Hence, we assumed a PK-target of $>2-4$ times the ECOFF of PSA $\left(f \mathrm{~T}_{>2-4}\right.$ ECOFF PSA $\left.=33-64 \mathrm{mg} / \mathrm{L}\right)$ as a rational compromise between effective bacterial killing and possibly harmful SCs. PIP concentrations of $65-99 \mathrm{mg} / \mathrm{L}$ were defined as moderately high. The definitions of sepsis/ severe sepsis and septic shock were based on the meanwhile revised SEPSIS-2 definition [27]. Patients were assigned to distinct BMI groups $\left(\leq 25,25-30,>30, \geq 40 \mathrm{~kg} / \mathrm{m}^{2}\right)$ to 
analyze effects on PIP-PK. Creatinine clearance (CrCL; mL/ min) was calculated using the Cockcroft-Gault formula [28]. The PIP clearance $\left(\mathrm{CL}_{\mathrm{PIP}} ; \mathrm{L} / \mathrm{h}\right)$ was derived using the following equation:

$\mathrm{CL}_{\mathrm{PIP}}\left[\mathrm{L} \mathrm{h}^{-1}\right]=\frac{\operatorname{dose}[\mathrm{mg}]}{24 \mathrm{~h}} \cdot c(\mathrm{PIP})^{-1}\left[\mathrm{mg} \mathrm{L}^{-1}\right]$

with c(PIP) being the measured PIP SC. In the absence of a universal definition, a CrCL of $\geq 130 \mathrm{~mL} / \mathrm{min}$ was defined as an augmented renal clearance (ARC) [29]. Five different PIP SC groups ( $<16,16-32,33-64,65-99$ and $\geq 100 \mathrm{mg} / \mathrm{L})$ were formed to depict SC distribution and to investigate the effects of PIP on outcome parameters.

\section{Statistical analysis}

PK-, TDM- and patient data were processed anonymously and included into a Microsoft Excel database (Microsoft Corp., Version 15.41). Statistical analysis was performed with IBM SPSS (IBM Corporation, Armonk, New York, Version 25). Descriptive statistics were reported as median (interquartile range (IQR); [range]), numbers and relative frequencies, or as means \pm standard deviations (SDs). Figures are given as box (median, IQR) and whisker (90th and 10th percentile) plots. Pearson correlation coefficients were used to elucidate correlations between clinical and PKrelated parameters. We used logistic regression analysis, variance analysis (ANOVA) and post hoc ANOVA where indicated to evaluate the effects of PK-target attainment. Kruskal-Wallis test, Pearson Chi square test and Fisher's $t$ test were performed to evaluate statistical significance. Significance was considered with $p \leq 0.05$.

\section{Results}

\section{Baseline epidemiological data and clinical characteristics}

Between December 2008 and October 2012, 484 patients (933 TDMs) were eligible for analysis (baseline characteristics given in Table 1). The 933 TDM measurements were mainly distributed over the first 6 days of treatment (day 1: $n=484$; day 2: $n=86$; day 3: $n=129$; day 4: $n=80$; day $5: n=58 ; \geq$ day $6: n=96$ ). The median PIP dose administered within the first $24 \mathrm{~h}$ of treatment was $12 \mathrm{~g}$ for all SC groups (<16 mg/L: $12 \mathrm{~g} ; 16-32 \mathrm{mg} / \mathrm{L}: 12 \mathrm{~g}$ (4 g;[2-16 g]); 33-64 mg/L: $12 \mathrm{~g} \mathrm{(4} \mathrm{g;[4-16} \mathrm{g]);>} 64 \mathrm{mg} / \mathrm{L}$ : $12 \mathrm{~g}(4 \mathrm{~g} ;[4-12 \mathrm{~g}]) .38 .6 \%$ of patients $(n=187 / 484)$ presented with sepsis or severe sepsis and $38.1 \%(n=184 / 484)$ with septic shock (Table 2). The primary sites of infection included lung/pneumonia $(n=256 / 484 ; 53 \%)$ and abdomen/
Table 1 Patient characteristics

\begin{tabular}{ll}
\hline$N(\%)$ & \\
Male & $301(62.2)$ \\
ARC & $41(8.5)$ \\
Mean $(S D)$ & \\
Age (years) & $73(17)$ \\
Height $(\mathrm{cm})$ & $170(12)$ \\
Weight $(\mathrm{kg})$ & $73(17)$ \\
Median $(I Q R)$ & \\
BMI $\left(\mathrm{kg} / \mathrm{m}^{2}\right)$ & $26.1(6.0)$ \\
Creatinine $(\mathrm{mg} / \mathrm{dL})$ & $1.22(1.26)$ \\
CrCL (mL/min) & $46.3(47.9)$ \\
Antimicrobial treatment (days) & $6.0(3.0)$ \\
Steady-state PIP serum concentration (mg/L) & $57.0(44.0)$ \\
PIP dose per 24 h (g) & $8.0(4.0)$ \\
\hline
\end{tabular}

Values given in absolute numbers $(N)$ and relative incidence $(\%)$, median and interquartile range (IQR) or mean and standard deviation of the mean (SD), respectively

$A R C$ augmented renal clearance, creatinine clearance $>130 \mathrm{~mL} / \mathrm{min}$, $B M I$ body mass index, $C r C L$ creatinine clearance, PIP PiperacillinTazobactam

Table 2 Baseline clinical data

\begin{tabular}{ll}
\hline Diagnosis & $N(\%)$ \\
\hline Sepsis/severe sepsis & $187(38.6)$ \\
Septic shock & $184(38.1)$ \\
Severe infection & $113(23.3)$ \\
Focus/site of infection & \\
Lung/pneumonia & $256(53)$ \\
Peritonitis & $114(23.6)$ \\
Urosepsis & $38(7.7)$ \\
SSTI & $17(3.5)$ \\
Empyema & $18(3.7)$ \\
BSI & $13(2.7)$ \\
Other & $28(5.6)$ \\
Renal replacement therapy & \\
Overall & $79(16.3)$ \\
CVVHD & $71(89.9)$ \\
iHD & $8(10.1)$ \\
Mechanical ventilation & \\
Overall & $317(65.5)$ \\
Duration & Median(IQR) \\
Mortality & $169(377)$ \\
ICU & \\
Hospital & $N(\%)$ \\
\hline
\end{tabular}

Values given in absolute numbers $(\mathrm{N})$ and relative incidence $(\%)$ or as median and interquartile range (IQR)

SSTI skin and soft tissue infection, BSI bloodstream infection, $C V V H D$ continuous veno-venous hemodialysis, $i H D$ intermittent hemodialysis, $I C U$ intensive care unit 
peritonitis $(n=114 / 484 ; 23.6 \%)$. Of the $16.3 \%(n=79 / 484)$ of patients receiving RRT, continuous veno-venous hemodialysis (CVVHD, citrate anticoagulation) was predominantly applied $(n=71 / 79 ; 89.9 \%)$ (Table 2$)$. Augmented renal clearance was detected in only $8.5 \%$ of patients $(n=41 / 484)$. Overall mortality rates were $19.2 \%(n=93 / 484)$ in the ICU and $23.2 \%(n=112 / 484)$ in hospital (Table 2$)$.

\section{Primary objectives}

\section{PK-target attainment within $24 \mathrm{~h}$ after treatment initiation}

The minimum PK-target of $\geq 33 \mathrm{mg} / \mathrm{L}$ was realized in $89.9 \%$ ( $n=435 / 484)$ of patients within $24 \mathrm{~h}$ of treatment whereas $34.3 \%(n=166 / 484)$ exactly met the designated PIP target range of 33-64 mg/L and 55.6\% $(n=269 / 484)$ exceeded the upper target of $64 \mathrm{mg} / \mathrm{L}$. Of these potentially overdosed patients, $25.4 \%(n=123 / 484)$ exhibited moderately high PIP SCs $(65-99 \mathrm{mg} / \mathrm{L})$. However, $30.2 \%(n=146 / 484)$ of patients showed considerably high PIP SCs of $\geq 100 \mathrm{mg} / \mathrm{L}$ that may increase the possibility of neuro- and/or nephrotoxicity with sustained exposure. Only $10.1 \%(n=49 / 484)$ of all patients showed low PIP SCs $(<16 \mathrm{mg} / \mathrm{L}, 0.2 \%$ [ $n=1 / 484]$, and $16-32 \mathrm{mg} / \mathrm{L}, 9.9 \%[n=48 / 484])$ after the initial dose (Fig. 1).

\section{Effects of TDM-guided dose adjustment measures}

Beyond the first $24 \mathrm{~h}, 449$ TDMs were performed to adjust PK-target attainment through customized PIP dosing. While the number of patients in the low PIP SC group did not considerably change $(<16 \& 16-32 \mathrm{mg} / \mathrm{L}: 15.8 \%, n=71 / 449)$, TDM-based dose adjustments profoundly increased the fraction of patients with PK-target attainment to $62 \%$ $(n=280 / 449)$ with median PIP SCs of $46 \mathrm{mg} / \mathrm{L}(16 \mathrm{mg} / \mathrm{L}$; [33-64 mg/L]), Table 3). Moreover, TDM-guided individual dosing reduced the fraction of patients with moderately elevated PIP SCs ( $\geq 64 \leq 100 \mathrm{mg} / \mathrm{L}$ ) by $32 \%$ to $17.4 \%$ ([n=78/449], Table 3$)$ and led to a major reduction of patients with potentially toxic concentrations $(\geq 100 \mathrm{mg} / \mathrm{L})$

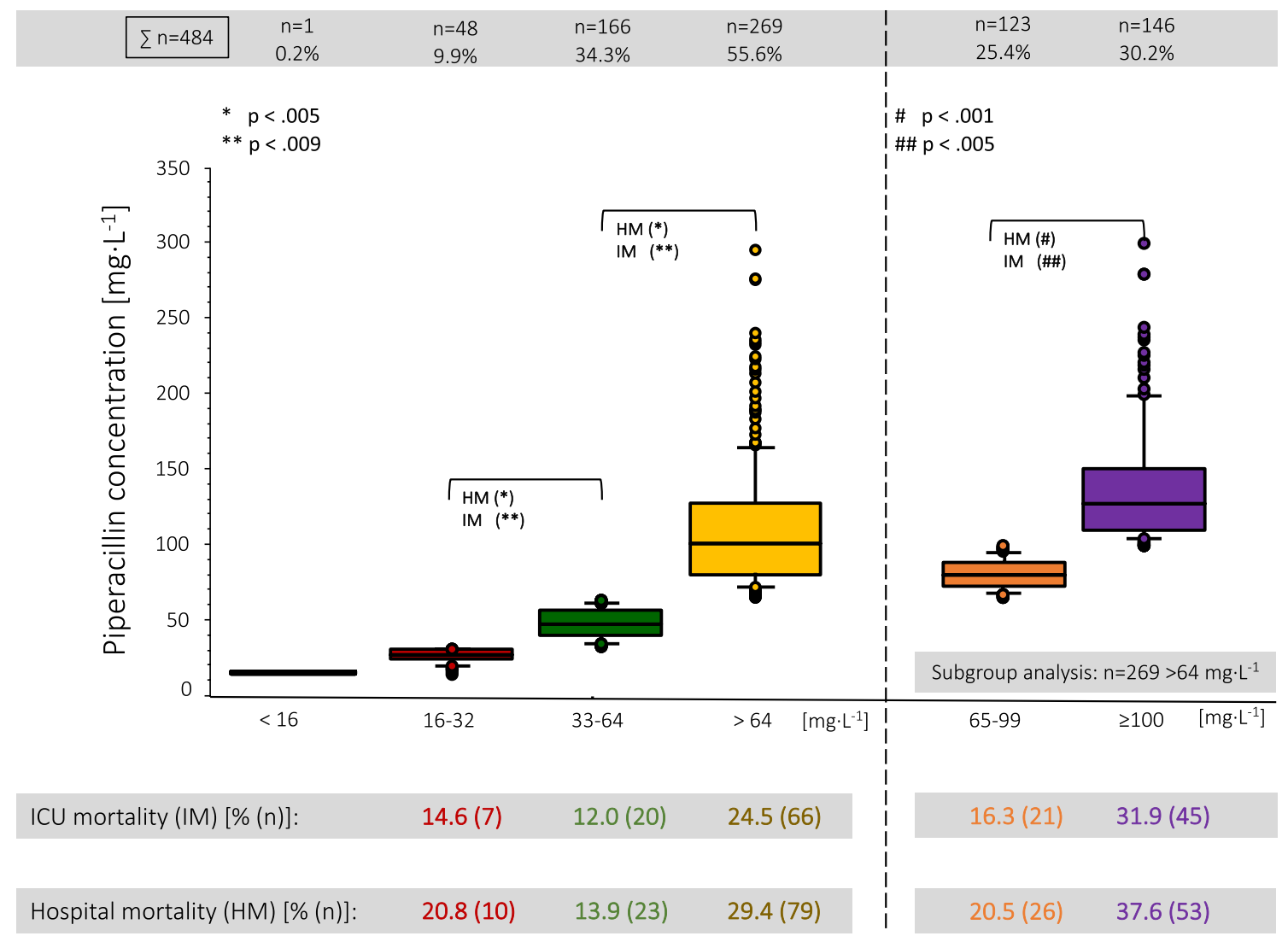

Fig. 1 Distribution of PIP serum concentrations within the first $24 \mathrm{~h}$ of treatment. Median PIP serum concentrations by group (median [IQR; range]): $16-32 \mathrm{mg} / \mathrm{L}$ (red): $29.0 \mathrm{mg} / \mathrm{L}$ [6.5 mg/L; 16-32 $\mathrm{mg} / \mathrm{L}]$. 33-64 $\mathrm{mg} / \mathrm{L}$ (green): $49.0 \mathrm{mg} / \mathrm{L} \quad[16.0 \mathrm{mg} / \mathrm{L}$; 33-64 mg/L]. 65-99 mg/L (orange): $80 \mathrm{mg} / \mathrm{L} \quad[16.0 \mathrm{mg} / \mathrm{L}$; 65-99 mg/L].> $100 \mathrm{mg} / \mathrm{L}$ (violet): $128 \mathrm{mg} / \mathrm{L}$ [41.0 mg/L; 100
$300 \mathrm{mg} / \mathrm{L}]$. Values given as absolute numbers $(\mathrm{N})$ and relative incidence $(\%)$ or median $(\mathrm{M})$, interquartile range (IQR) and range (minimum to maximum value). Statistical analysis was assessed using cross tables and $\mathrm{Chi}^{2}$ test. Significance level 5\% $(p<0.05)$. IM intensive care unit mortality, $H M$ hospital mortality, $I Q R$ interquartile range 
Table 3 Effect of TDM-guided dose adjustment measures on PK-target attainment

\begin{tabular}{llllllll}
\hline & & \multicolumn{1}{c}{ Subgroup $>64 \mathrm{mg} / \mathrm{L}$} \\
\hline PIP SC (mg/L) & & $<16$ & $16-32$ & $33-64$ & $>64$ & $65-99$ & $\geq 100$ \\
24 h, \% $(N)$ & $n=484$ & $0.2(1)$ & $9.9(48)$ & $34.3(166)$ & $55.6(269)$ & $25.4(123)$ & $30.2(146)$ \\
TDM-guided, \% $(N)$ & $n=449$ & $1.1(5)$ & $14.7(66)$ & $62.4(280)$ & $21.8(98)$ & $17.4(78)$ & $4.5(20)$ \\
\hline
\end{tabular}

Comparison of PK-target attainment within the first $24 \mathrm{~h}(n=484)$ of treatment and after TDM-guided customized PIP dosing in the course of treatment ( $n=449$ TDMs)

Values given in absolute numbers $(N)$ and relative incidence $(\%)$

PIP SC Piperacillin serum concentration, TDM therapeutic drug monitoring, $P K$ pharmacokinetics

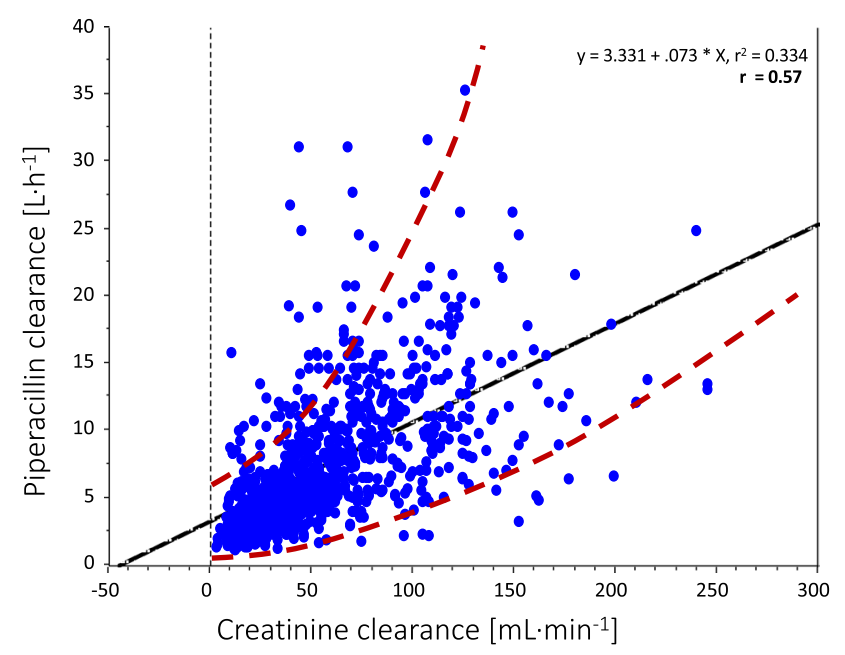

Fig. 2 Association of $\mathrm{CrCL}$ and $\mathrm{CL}_{\mathrm{PIP}}$. Correlation of renal function (CrCL) and $\mathrm{CL}_{\mathrm{PIP}}$ in the analyzed cohort of ICU patients. Values given as absolute numbers. Correlation was assessed by calculating a Pearson correlation coefficient. $C r C L$ creatinine clearance, $C L_{P I P}$ piperacillin clearance

by $85-4.5 \%$ ( $n=20 / 449$, Table 3$)$. Overall, TDM effectively enhanced target attainment while reducing potential toxic PIP SCs at the same time.

\section{Secondary objectives: effects of renal function and RRT on PIP-PK}

With a coefficient of $r=0.57$, overall correlation of CrCL and $\mathrm{CL}_{\mathrm{PIP}}$ was good and comparable to existing literature [30-32]. However, Fig. 2 clearly shows increasing variation of $\mathrm{CL}_{\mathrm{PIP}}$ with $\mathrm{CrCL}$ values of above $80-100 \mathrm{~mL} / \mathrm{min}$. This finding demonstrates the difficulty of predicting drug clearance of PIP as a sole function of CrCL in critically ill patients, particularly in those with sepsis and septic shock. Binary logistic regression further revealed high CrCL to significantly increase the odds for insufficiently low PIP SCs within the investigated study cohort $(<16 \mathrm{mg} / \mathrm{L}: p<0.0005$; OR $1.02295 \%$ CI [1.011-1.034] and 16-32 mg/L: $p<0.0005$; OR 1.017 95\% CI [1.013-1.022]). These data highlight the role of renal function on drug clearance.
Particularly, increased renal function can be presumed as a main risk factor for target non-attainment.

Regardless of the technique used, RRT in general was accompanied by a marked, however not significant, reduction of $\mathrm{CL}_{\mathrm{PIP}}$ (Fig. 3a). Although the median PIP dose did not profoundly change (CVVHD: $8 \mathrm{~g}$ (2 g; [3-12 g]) vs iHD:

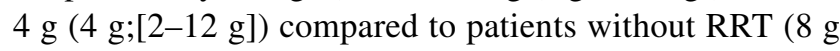
(4 g;[3-20 g]), evaluation of the respective boxplots showed a clear tendency to lower doses and less pronounced variations during RRT (Fig. 3b). Interestingly, although CL $_{\text {PIP }}$ during RRT and PIP doses applied was lower during RRT, PIP SCs in this group were strikingly higher compared to non-RRT patients. CVVHD was associated with an $46.3 \%$ increase $(98.0 \mathrm{mg} / \mathrm{L}(63 \mathrm{mg} / \mathrm{L} ;[21-244 \mathrm{mg} / \mathrm{L}])$ and iHD appeared to account for an elevation of PIP SCs by $51.7 \%$ (129.5 mg/L (76.5 mg/L;[75-240 mg/L]).

\section{Effect of BMI on PIP-PK}

In our study cohort, the group of morbidly obese (patients $\geq 40 \mathrm{~kg} / \mathrm{m}^{2}, n=18 / 484$ [3.7\%], SC $n=43 / 933$ [4.6\%]) patients showed a significantly higher $\mathrm{CL}_{\mathrm{PIP}}(9.6$ $\mathrm{L} / \mathrm{h}(7.7 \mathrm{~L} / \mathrm{h} ;[1.4-27.8 \mathrm{~L} / \mathrm{h}]) ; p<0.0001)$ compared to all other BMI groups (Fig. 4). As increased renal blood flow is discussed to contribute to elevated $\mathrm{CL}_{\mathrm{PIP}}$ in obese patients [33], we assessed and compared CrCL in the different BMI groups. Interestingly, morbidly obese patients did not show significantly higher values for CrCL as compared to other BMI groups (Fig. 5). Our data do not support that an increase in renal clearance accounts for elevated $\mathrm{CL}_{\mathrm{PIP}}$ in morbidly obese patients.

\section{Effect of target attainment on ICU- and hospital mortality} (Fig. 1, Table 4)

ICU mortality (12.0\% [33-64 mg/L] vs. 14.6 [16-32 mg/L], $16.3[65-99 \mathrm{mg} / \mathrm{L}]$ and $31.5 \%[\geq 100 \mathrm{mg} / \mathrm{L}] ; p=0.009)$ and hospital mortality $(13.9 \%$ [33-64 mg/L] vs. 20.8 [16-32 mg/L], 20.5 [65-99 mg/L] and 37.6\% [ $\geq 100 \mathrm{mg} / \mathrm{L}]$; $p=0.005)$ were significantly lower in patients with exposure to the defined PK-target concentration of 33-64 mg/L within the first 24 h (Fig. 1). Exposure to PIP 
A
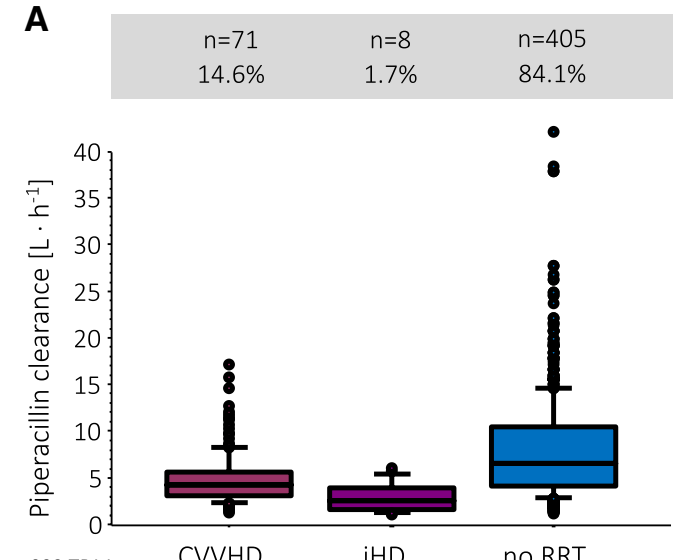

$n=933$ TDM

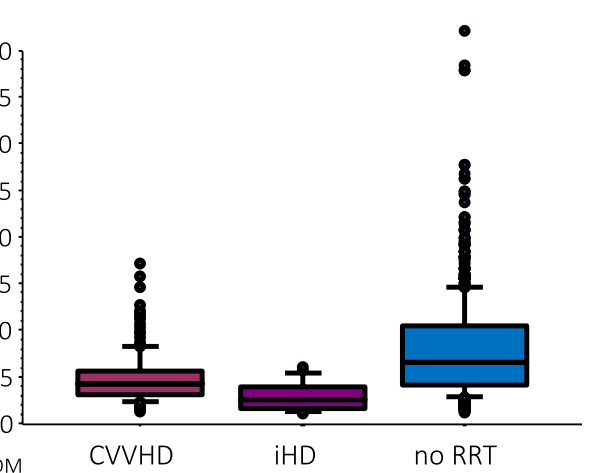

Fig. 3 Effects of RRT. A: Influence of RRT (89.9\% CVVHD, 10.1\% iHD) on $\mathrm{CL}_{\mathrm{PIP}}$ compared to the group of patients without RRT. $\mathrm{C}_{\mathrm{LPIP}}$ by RRT mode (median [IQR; range]): CVVHD (magenta): 4.3 $\mathrm{L} / \mathrm{h}[2.6 \mathrm{~L} / \mathrm{h} ; 1.4-17.2 \mathrm{~L} / \mathrm{h}]$. iHD (violet): $2.6 \mathrm{~L} / \mathrm{h}[2.3 \mathrm{~L} / \mathrm{h} ; 1.2-6.2$ L/h]. no RRT (blue): $6.8 \mathrm{~L} / \mathrm{h}[6.3 \mathrm{~L} / \mathrm{h} ; 1.3-35.5 \mathrm{~L} / \mathrm{h}]$. B: PIP doses administered to to patients with RRT (magenta\&violet) compared to

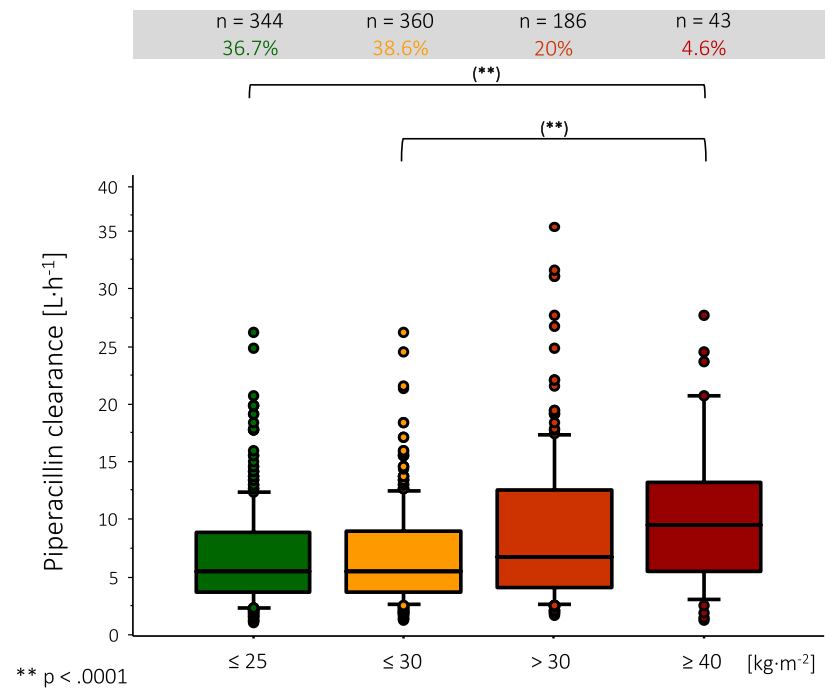

Fig. 4 Distribution of $\mathrm{CL}_{\mathrm{PIP}}$ according to different BMI groups. $\mathrm{CL}_{\mathrm{PIP}}$ by BMI group (median [IQR; range]): $\leq 25 \mathrm{~kg} / \mathrm{m}^{2}$ (green): $5.6 \mathrm{~L} / \mathrm{h}$ [5.1 L/h; 1.2-26.3 L/h]. $\leq 30 \mathrm{~kg} / \mathrm{m}^{2}$ (yellow): $6.9 \mathrm{~L} / \mathrm{h}[5.3 \mathrm{~L} / \mathrm{h} ; 1.4-$ $26.3 \mathrm{~L} / \mathrm{h}] .>30 \mathrm{~kg} / \mathrm{m}^{2}$ (red): $6.9 \mathrm{~L} / \mathrm{h}[8.5 \mathrm{~L} / \mathrm{h} ; 1.8-35.5 \mathrm{~L} / \mathrm{h}] . \geq 40 \mathrm{~kg} /$ $\mathrm{m}^{2}$ (dark red): $9.6 \mathrm{~L} / \mathrm{h}[7.7 \mathrm{~L} / \mathrm{h} ; 1.4-27.8 \mathrm{~L} / \mathrm{h}]$. Values given as absolute numbers $(N)$ and relative incidence $(\%)$ or median $(\mathrm{M})$, interquartile range (IQR) and range (minimum to maximum value), respectively. Statistical analysis was performed using analysis of variance \& post hoc analysis with Bonferroni/Dunn correction. Significance level $5 \%(p<0.05)$. PIP piperacillin, $C L_{P I P}$ PIP clearance, $B M I$ body mass index, $I Q R$ interquartile range

concentrations $\geq 100 \mathrm{mg} / \mathrm{L}$ resulted in significantly higher mortality (ICU mortality: $31.9 \%, n=45 / 484, p=0.005$; hospital mortality: $37.6 \%, n=53 / 484, p=0.001$ ) compared to the PK-target group and patients with moderately high
B

$\begin{array}{ccc}n=71 & n=8 & n=405 \\ 14.6 \% & 1.7 \% & 84.1 \%\end{array}$

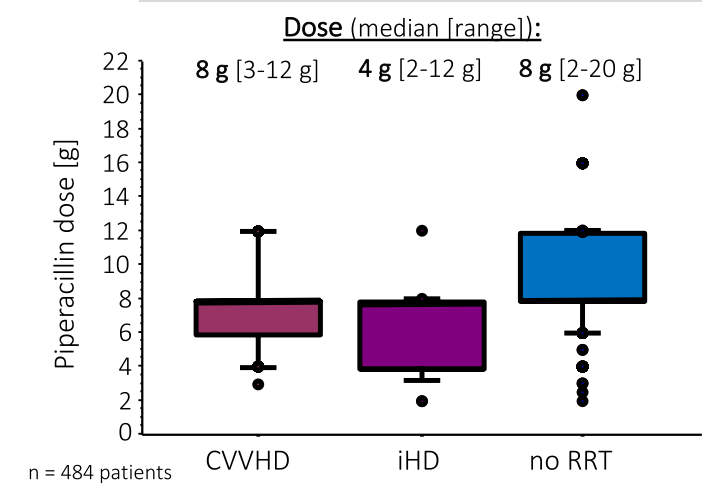

the non-RRT group (blue). Values given as absolute numbers $(N)$ and relative incidence $(\%)$ or median $(\mathrm{M})$, interquartile range (IQR) and range (minimum to maximum value), respectively. $R R T$ renal replacement therapy, $C V V H D$ conitnuous veno-venous hemodialysis, $i H D$ intermittent hemodialysis, CLPIP piperacillin clearance

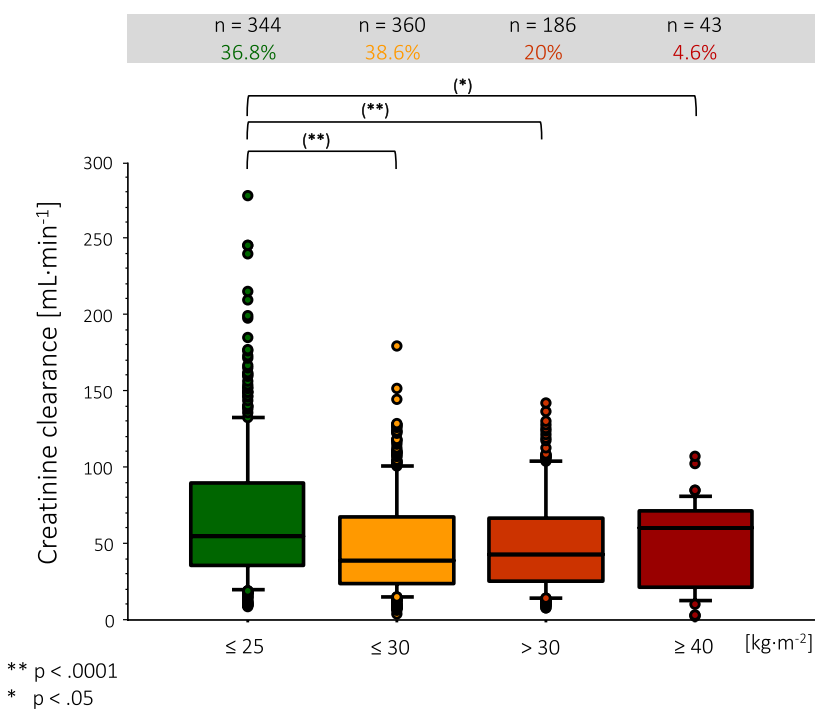

Fig. 5 Distribution of $\mathrm{CrCL}$ according to BMI. CrCL by BMI group (median [IQR; range]): $\leq 25 \mathrm{~kg} / \mathrm{m}^{2}$ (green): $55.8 \mathrm{~mL} / \mathrm{min}[54.5 \mathrm{~mL} /$ min; $9.0-277.9 \mathrm{~mL} / \mathrm{min}] . \leq 30 \quad \mathrm{~kg} / \mathrm{m}^{2}$ (yellow): $39.3 \mathrm{~mL} / \mathrm{min}$ [43.2 $\mathrm{mL} / \mathrm{min} ; 4.3-179.5 \mathrm{~mL} / \mathrm{min}] .>30 \mathrm{~kg} / \mathrm{m}^{2}$ (red): $42.8 \mathrm{~mL} / \mathrm{min}$ [41.7 mL/min; 7.6-141.8 mL/min]. $\geq 40 \mathrm{~kg} / \mathrm{m}^{2}$ (dark red): $60.1 \mathrm{~mL} /$ min $[49.8 \mathrm{~mL} / \mathrm{min} ; 2.5-107.0 \mathrm{~mL} / \mathrm{min}]$. Values given as absolute numbers $(N)$ and relative incidence $(\%)$ or median $(\mathrm{M})$, interquartile range (IQR) and range (minimum to maximum value), respectively. Statistical analysis was performed by analysis of variance \& post hoc analysis with Bonferroni/Dunn-correction. Significance level 5\% $(p<0.05) . C r C L$ creatinine clearance, $C L_{P I P}$ piperacillin clearance

(65-99 mg/L) PIP SCs. Moreover, we separately analyzed the mortality in the subgroup of patients with exposure to SCs between 100-160 $(n=40 / 115)$ and $>160 \mathrm{mg} / \mathrm{L}$ 
Table 4 Cross table depicting the distribution of clinical parameters in different PIP serum concentration group

\begin{tabular}{llllll}
\hline PIP SC $[\mathrm{mg} / \mathrm{L}]$ & $33-64$ & $\leq 16$ & $16-32$ & $65-99$ & $\geq 100$ \\
\hline$N(\%) ; p$ value & & & & & $\sum N$ \\
$\quad$ Severe $)$ sepsis & $69(41.3)$ & & $26(54.2) ; .09$ & $43(37.4) ; .121$ & $49(31.8) ; .080$ \\
Septic Shock & $49(29.3)$ & & $12(25) ; .347$ & $48(41.7) ; 0.049$ & $76(49.4) ;<0.0005$ \\
Ventilation & $104(63.3)$ & $1(0.3)$ & $31(64.6) ; .504$ & $80(65.3) ; .410$ & $101(69.2) ; .163$ \\
RRT & $15(9.0)$ & & $4(8.2) ; .576$ & $21(16.5) ; 0.019$ & $39(26.6) ;<0.0005$ \\
Median (IQR; min-max); $p$ value & & & & 317 \\
Age (years) & $70(17.8 ; 17-92)$ & 44 & $59.5(24 ; 19-88) ;<0.0005$ & $74(14.5 ; 21-91)$ & $75.5(12.0 ; 16-91)<0.0005$ \\
CrCL (mL/min) & $61.3(50 ; 10.7-245.2)$ & 125.5 & $79.1(65.8 ; 9.7-179.5) ;$ & $40.9(37.6 ; 7.6-176.8) .119$ & $26.9(24.9 ; 2.9-152.1)<$ \\
& & & 0.011 & & 0.0005 \\
\hline
\end{tabular}

Values given as absolute numbers $(N)$ and relative incidence $(\%)$ or median $(\mathrm{M})$ and interquartile range (IQR; Min.-Max.), respectively. Statistical analysis was assessed using $\mathrm{Chi}^{2}$ - and Mann-Whitney $U$ test. Significance level $5 \%(p<0.05)$

$P I P$ Piperacillin, $S C$ serum concentration, $R R T$ renal replacement therapy, $I C U$ intensive care unit, $C r C L$ creatinine clearance, $I Q R$ interquartile range

( $n=15 / 31)$. Both subgroups showed strikingly high ICU $(30.4 \%$ and $35.5 \%)$ and hospital mortality rates $(34.8 \%$ and $48.4 \%$ ). In the group of patients with $\mathrm{SC}>160 \mathrm{mg} / \mathrm{L}$, we observed the highest median (IQR) PIP dose 12000 (4000) $\mathrm{mg}$, the highest SC 192 (56.3) $\mathrm{mg} / \mathrm{L}$ and the lowest CrCL 21.7 (20.6) $\mathrm{mL} / \mathrm{min}$. Overall, we found a U-shaped distribution of mortality rates depending on the PIP SCs measured. We evaluated CrCL and SCs of survivors and deceased patients and found deceased to have significantly lower CrCL (31.8 mL/min (31.1 mL/min;[2.9-159 mL/ $\min ]), p<0.0001)$ as compared to survivors $(48.5 \mathrm{~mL} /$ $\min (48.5 \mathrm{ml} / \mathrm{min}$; [4.3-245.2 $\mathrm{mL} / \mathrm{min}])$ and significantly higher PIP SCs $(98.5 \mathrm{mg} / \mathrm{L}(75 \mathrm{mg} / \mathrm{L} ;[21-244 \mathrm{mg} / \mathrm{L}])$ vs. $66.5 \mathrm{mg} / \mathrm{L}$ (53 mg/L;[14.1-300 mg/L], $p<0.0001)$.

\section{Discussion}

To the best of our knowledge, this is the largest reported experience of routinely used TDM-guided CI of PIP and customized PIP dosing. Positive effects of CI of $\beta$-lactams on patient outcome have been repeatedly reported $[17,19$, 22, 34]. An important metanalysis by Roberts et al. [18] with remarkably low heterogeneity $\left(I^{2}=0\right.$ for hospital and ICU mortality) including 632 patients strikingly demonstrated significantly lower 30-day hospital mortality using CI of $\beta$-lactams.

We realized the minimum $\mathrm{PK}$-target of $\geq 33 \mathrm{mg} / \mathrm{L}$ in $89.9 \%(n=435 / 484)$ of patients within $24 \mathrm{~h}$ of treatment whereas $34.3 \%(n=166 / 484)$ exactly met the designated PIP target range of 33-64 mg/L. TDM-guided dose adjustments could significantly enhance PK-target attainment (33-64 $\mathrm{mg} / \mathrm{L})$ by $81.9 \%$ and at the same time effectively reduced the number of patients with potentially harmful concentrations of $\geq 100 \mathrm{mg} / \mathrm{L}$ by $85 \%$. Our data do not support previous findings of insufficiently low SCs [9, 35, 36] associated with CI of $\beta$-lactams. Underdosing during CI of PIP did rarely occur in the evaluated study cohort $(\leq 24 \mathrm{~h}$ : $10.1 \%, n=49 / 484 ;>24$ h: $15.8 \%, n=71 / 449)$. In contrast to our findings, a recent prospective study of Dhaese et al. [36] found a remarkably high PK-target non-attainment in ICU patients enrolled to receive a CI of PIP (62.9\% target non-attainment) or meropenem (25\% target non-attainment). A possible explanation for the poor target attainment might be the higher PK-target of $100 \% f \mathrm{~T}_{>4 \text { ECOFF }}$ of PSA ( $\geq 64-160 \mathrm{mg} / \mathrm{L}$ ) itself. Although we used a lower PK-target in our study, PIP SCs $\geq 100 \mathrm{mg} / \mathrm{L}$ occurred in $30.2 \%$ ( $n=146 / 484$ ) of patients within the first $24 \mathrm{~h}$ of treatment. We would like to argue that main contributing factors for the divergent target attainment between Dhaese and our study are in fact the different PK-targets and the significantly different study population. Dhaese et al. [36] included younger patients that rarely required vasopressor support which is uncommon in critically ill patients. Our patients are almost 10 years older and the estimated median [IQR] CrCL is more compromised $(46.3 \mathrm{~mL} / \mathrm{h}$ [47.9 $\mathrm{mL} / \mathrm{h}])$ as compared to the ICU cohort of Dhaese and coworkers (Pip/Taz $95.4 \mathrm{~mL} / \mathrm{h}$ [58.3 mL/h]; Mero $117.8 \mathrm{~mL} / \mathrm{h}$ [68.4 mL/h]). Moreover, APACHE II and SOFA scores were only moderately high and patients with RRT were excluded. Our study cohort comprised $76.6 \%$ of patients with sepsis and septic shock including patients with RRT (Table 4). The PK-target (100\% $\left.f \mathrm{~T}_{>4 \mathrm{xECOFF}},>64 \leq 160 \mathrm{mg} / \mathrm{L}\right)$ defined by Dhaese et al. [36] may be justified in a worst-case-scenario (PSA with MIC 16). EUCAST data show that only $11 \%$ of PSA have an MIC of 16. Most of the strains exhibit an MIC of 0.5-8 (https://mic. eucast.org/Eucast2/regShow.jsp?Id=6919). Consequently, we defined a lower primary PK-target $\left(100 \% f \mathrm{~T}_{>2-4 x E C O F F}\right.$, 33-64 mg/L) to balance effective bacterial killing and possibly harmful side effects. Our data highlight a U-shaped association of PIP SCs and mortality (Fig. 1). Exposure to higher than defined SCs was associated with significantly higher 
mortality rates (especially $\mathrm{SCs} \geq 100 \mathrm{mg} / \mathrm{L}$ ), especially in patients with exposure to PIP SCs of 100-160 (hospital mortality $35.5 \%$, ICU mortality $30.4 \%)$ and $>160 \mathrm{mg} / \mathrm{L}(48.4 \%$, $34.8 \%)$. We observed the highest survival rate in the patients with target attainment (33-64 mg/L), whereas Dhaese et al. [34] demonstrated a significantly lower survival rate in the group of patients with target attainment. In addition, these patients demonstrated a significantly lower CrCL.

With regard to adverse effects, Imani et al. [37] demonstrated neuro- and nephrotoxicity during bolus application of PIP. Quinton et al. [38] predicted neurotoxicity following continuous application of PIP at around a threshold concentration of $157 \mathrm{mg} / \mathrm{L}$ with a sensitivity of $52 \%$ which implies that neurotoxicity might well happen above and below $157 \mathrm{mg} / \mathrm{L}$ that concentration. Noticeably, patients with neurotoxic symptoms showed a significantly lower eGFR $(18 \mathrm{~mL} / \mathrm{min})$ as compared to those without neurotoxicity $(50 \mathrm{~mL} / \mathrm{min})$. The patients with neurotoxicity $(n=23)$ reached significantly higher PIP SCs $(156 \mathrm{mg} / \mathrm{L})$ and demonstrated a relevant reduction of GFR by approximately $45 \%$ $(33 / 18 \mathrm{~mL} / \mathrm{min})$ within 3 days of antibiotic treatment. In contrast, the rest of the cohort demonstrated an increase of GFR by $13 \%$ (44/50 $\mathrm{mL} / \mathrm{min})$. On the day of PIP SC measurements, the dose normalized to eGFR $(\mathrm{g} / 24 \mathrm{~h} / 100 \mathrm{~mL} /$ $\min / 1.73 \mathrm{~m}^{2}$ ) was significantly higher in the neurotoxicity group as compared to the rest of the group (48 [35.3-69.7] versus 22 [14.3-54]; $p=0.0111$ ). We do think that this strongly hints to a potentially harmful effect in patients with very high PIP SCs (>160 mg/L) and supports our finding that PIP SCs considerably higher than 100 and $160 \mathrm{mg} / \mathrm{L}$ may in fact be detrimental for kidney function in critically ill patients.

In accordance with previous data [30], we identified age and $\mathrm{CrCL}$ as two important factors for PK-target non-attainment in our study cohort: the odds for low PIP SCs significantly increased with high CrCL $(<16 \mathrm{mg} / \mathrm{L}$ : OR 1.002 95\% CI [1.011-1.034]; 16-32 mg/L: OR 1.017 95\% CI [1.013-1.022]; $p<0.0005)$ while age significantly increased the odds for PIP SCs $\geq 100 \mathrm{mg} / \mathrm{L}$ (OR $1.04495 \%$ CI [1.029-1.060]; $p<0.0005)$. Intensivists need to recognize that exposure to PK-targets impacts treatment success and patient outcome. Higher age and ARC are relevant risk factors for PK-target non-attainment. In that sense, our data are an important contribution to previous studies $[35,39]$ stating that customized PIP dosing [24] is the only reliable way to enhance PK-target attainment, preventing over- and underdosing and thereby presumably improving patient outcome.

As illustrated in Table 4, impaired renal function (and PIP elimination) may cause high SCs but vice versa, high PIP SCs may readily exacerbate a preexisting renal dysfunction resulting in acute kidney injury with RRT. The potentially nephrotoxic effects of PIP are of special concern when a combination therapy (i.e., vancomycin) is pursued. Recent data clearly illustrate a higher incidence of acute kidney injury in combination therapy and furthermore a distinct effect of piperacillin [40-42]. Recent data [37] further support the notion that high piperacillin serum concentrations alone may be associated with nephrotoxicity; at least when administered as intermittent bolus application. Patients who developed nephrotoxic signs demonstrated mean PIP trough concentrations $>100 \mathrm{mg} / \mathrm{L}$ and those without any signs showed concentrations considerably $<100 \mathrm{mg} / \mathrm{L}$.

As one of only few studies we specifically investigated the effect of RRT on PIP-PK during CI. Consistent with other data [43], we found a considerable reduction of $\mathrm{CL}_{\mathrm{PIP}}$ during RRT with markedly higher PIP SCs compared to non-RRT patients. One explanation may be the constant and renalindependent elimination of PIP during. In patients with PIP SCs of $\geq 100 \mathrm{mg} / \mathrm{L}$, we found significantly more patients with acute renal failure requiring RRT. Vice versa, potentially toxic PIP concentrations might as well exacerbate preexisting renal dysfunction and attribute to acute kidney injury [37]. CVVHD is the most commonly used form of RRT in critically ill patients $[44,45]$ but it is far from being a standardized system. Different modes of operation, variations in flow and the type of hemofilter used may influence $\mathrm{CL}_{\mathrm{PIP}}$ and PIP-PK [46-49].

Although pathophysiologic changes in obese patients [50, 51] may impose dosing problems, our data do not support this notion in the context of CI of PIP. The significantly higher $\mathrm{CL}_{\mathrm{PIP}}$ not associated with increased renal drug clearance in patients with a BMI $\left(\geq 40 \mathrm{~kg} / \mathrm{m}^{2}\right)$ might hint to increased extra-renal drug clearance, i.e., through the gastrointestinal tract and/or drug deposition in fatty or muscle tissue. A significantly lower target attainment $\left(f T_{>4 \times M I C}\right)$ in obese patients has been previously demonstrated presumably as a drug-specific effect of piperacillin [52]. Therefore, these data emphasize the rationale for TDM particularly in obese patients in the ICU and the necessity for further trials investigating PIP and $\beta$-lactam PK alterations in this subgroup of patients [53-55].

Our findings underscore the need for definite studies investigating the effects of PK-target attainment along with CI of $\beta$-lactams on mortality. The ongoing TARGET study [56], investigating TDM-based dose optimization of piperacillin/tazobactam to improve outcome in patients with sepsis, will particularly address target attainment and CI. The aspect of CI of $\beta$-lactams will be re-assessed in the BLING III-study, an ongoing phase 3 multicenter randomized controlled trial investigating continuous versus intermittent $\beta$-lactam antibiotic infusion in critically ill patients with sepsis [57]. Both studies will also improve our understanding of the PK alterations of $\beta$-lactams in critically ill patients.

Our study has several limitations. The data were analyzed retrospectively and drawn from a single center where 
$\beta$-lactams have been routinely and solely been administered as a TDM-guided CI for more than 10 years. In the absence of control groups and possible confounding, mortality data must be interpreted prudentially. Even though we report a large number of routine TDM measurements, correlation with clinical scores [Sepsis-related Organ Failure Assessment Score (SOFA), Simplified Acute Physiology Score (SAPS)] was not possible. Microbiological data and MICs of pathogens were not available for analysis. HPLC was performed to measure PIP concentrations while the combination of PIP and tazobactam (PIP/TAZ) was administered to the patients. Recent evidence, however, suggests that the PK alterations of both PIP and TAZ are almost equal in healthy adults [58] and critically ill patients $[59,60]$. $\beta$-lactamase inhibition with tazobactam occurs rapidly, within 20-30 min [58] and additionally, piperacillin inhibits tubular excretion of tazobactam [61].

\section{Conclusion}

Our data strongly support the use of TDM-guided CI of PIP in critically ill patients. This customized approach leads to sufficient PK-target attainment within the first hours of treatment while critically low PIP SCs rarely occurred. To the best of our knowledge, this is the largest reported experience of routinely used TDM-guided CI of PIP to report the effect of CI on PK-target attainment as well as PK changes with regard to renal function/RRT and obesity. We further found evidence of a reduction of mortality by achieving predefined target SC facilitating adequate exposures of PIP in critically ill with severe infections, sepsis and septic shock. The results are only valid for continuous application of piperacillin/tazobactam.

\section{Compliance with ethical standards}

Conflict of interest DC.R. has received refunding of travel expenses from Gilead and Astellas Pharma. O.F. has no conflicts. A.R. has no conflicts. J.A.R. has served as a consultant/lecturer for MSD, Bayer, Astellas, bioMerieux and Accelerate Diagnostics and held investigator-initiated grants from MSD and The Medicines Company. A.K. has no conflicts. T.F. has no conflicts. N.P. has no conflicts. C.L. has no conflicts. T.B. has received lecture fees and/or refunding of travel expenses from: Baxter, Schöchl medical education, Boehringer Ingelheim Pharma, CSL Behring, Astellas Pharma, B. Braun Melsungen, MSD Sharp \& Dohme and is member of advisory boards for: Baxter \& Biotest. M.A.W. has received lecture fees and/or refunding of travel expenses from: Astellas Pharma, Astra Zeneca, B.Braun, Biosyn, CLS Behring, Cytosorb, Eli Lilly, GE Healthcare, Gilead, Glaxo Smith Kline, Janssen, Köhler Chemie, MSD Sharp \& Dohme, Novartis, Orion \& Pfizer Pharma and is member of advisory boards for: Astellas Pharma, B.Braun, Gilead, MSD Sharp \& Dohme, Pall Medical \& Pfizer Pharma. A.B. has received lecture fees and/or refunding of travel expenses from: Grünenthal GmbH, Pfizer Pharma GmbH, Fresenius Medical Care (FMC), Niedersächsisches Landesgesundheitsamt,
LADR-Laboratory Bremen, Laborbetriebsgesellschaft Dr. Dirkes-Kersting $\mathrm{GmbH}$, Gelsenkirchen, Laboratory Volkmann, Karlsruhe.

Open Access This article is distributed under the terms of the Creative Commons Attribution 4.0 International License (http://creativeco mmons.org/licenses/by/4.0/), which permits unrestricted use, distribution, and reproduction in any medium, provided you give appropriate credit to the original author(s) and the source, provide a link to the Creative Commons license, and indicate if changes were made.

\section{References}

1. Rhodes A, Evans LE, Alhazzani W, Levy MM, Antonelli M, Ferrer R, et al. Surviving sepsis campaign: international guidelines for management of sepsis and septic shock. Intensive Care Med. 2016;2017:1-74.

2. Kumar A, Roberts D, Wood KE, Light B, Parrillo JE, Sharma S, et al. Duration of hypotension before initiation of effective antimicrobial therapy is the critical determinant of survival in human septic shock. Crit Care Med. 2006;34(6):1589-96.

3. Ferrer R, Martin-Loeches I, Phillips G, Osborn TM, Townsend S, Dellinger RP, et al. Empiric antibiotic treatment reduces mortality in severe sepsis and septic shock from the first hour: results from a guideline-based performance improvement program. Crit Care Med. 2014;42(8):1749-55.

4. Kumar A, Ellis P, Arabi Y, Roberts D, Light B, Parrillo JE, et al. Initiation of inappropriate antimicrobial therapy results in a fivefold reduction of survival in human septic shock. Chest. 2009;136(5):1237-48.

5. Magill SS, Edwards JR, Bamberg W, Beldavs ZG, Dumyati G, Kainer MA, et al. Multistate point-prevalence survey of health care-associated infections. N Engl J Med. 2014;370(13):1198-208.

6. Roberts JA, Abdul-Aziz MH, Lipman J, Mouton JW, Vinks AA, Felton TW, et al. Individualised antibiotic dosing for patients who are critically ill: challenges and potential solutions. Lancet Infect Dis. 2014;14(6):498-509.

7. Brinkmann A, Röhr A, Köberer A, Fuchs T, Krüger W, König C, et al. Adäquate Antiinfektivatherapie. Der Anaesthesist. 2018:1-15.

8. Richter D, Heininger A, Brenner T, Hochreiter M, Bernhard M, Briegel J, et al. Bakterielle sepsis. Der Anaesthesist. 2017;66(10):737-61.

9. Roberts JA, Paul SK, Akova M, Bassetti M, De Waele JJ, Dimopoulos G, et al. DALI: defining antibiotic levels in intensive care unit patients: are current beta-lactam antibiotic doses sufficient for critically ill patients? Clin Infect Dis. 2014;58(8):1072-83.

10. Abdul-Aziz MH, Sulaiman H, Mat-Nor MB, Rai V, Wong KK, Hasan MS, et al. Beta-Lactam Infusion in severe sepsis (BLISS): a prospective, two-centre, open-labelled randomised controlled trial of continuous versus intermittent beta-lactam infusion in critically ill patients with severe sepsis. Intensive Care Med. 2016;42(10):1535-45.

11. De Waele JJ, Carrette S, Carlier M, Stove V, Boelens J, Claeys $\mathrm{G}$, et al. Therapeutic drug monitoring-based dose optimisation of piperacillin and meropenem: a randomised controlled trial. Intensive Care Med. 2014;40(3):380-7.

12. Zander J, Döbbeler G, Nagel D, Maier B, Scharf C, Huseyn-Zada $\mathrm{M}$, et al. Piperacillin concentration in relation to therapeutic range in critically ill patients-a prospective observational study. Crit Care. 2016;20(1):79. 
13. Craig WA. Pharmacokinetic/pharmacodynamic parameters: rationale for antibacterial dosing of mice and men. Clin Infect Dis. 1998;26(1):1-10.

14. Gonçalves-Pereira J, Póvoa P. Antibiotics in critically ill patients: a systematic review of the pharmacokinetics of $\beta$-lactams. Crit Care. 2011;15(5):1.

15. Dulhunty JM, Roberts JA, Davis JS, Webb SA, Bellomo R, Gomersall $\mathrm{C}$, et al. Continuous infusion of beta-lactam antibiotics in severe sepsis: a multicenter double-blind, randomized controlled trial. Clin Infect Dis. 2013;56(2):236-44.

16. Abdul-Aziz MH, Lipman J, Akova M, Bassetti M, De Waele JJ, Dimopoulos G, et al. Is prolonged infusion of piperacillin/ tazobactam and meropenem in critically ill patients associated with improved pharmacokinetic/pharmacodynamic and patient outcomes? An observation from the Defining Antibiotic Levels in Intensive care unit patients (DALI) cohort. J Antimicrob Chemother. 2016;71(1):196-207.

17. Rhodes NJ, Liu J, o'donnell JN, Dulhunty JM, Abdul-Aziz MH, Berko PY, et al. Prolonged infusion piperacillin-tazobactam decreases mortality and improves outcomes in severely ill patients: results of a systematic review and meta-analysis. Crit Care Med. 2018;46(2):236-43.

18. Roberts JA, Abdul-Aziz M-H, Davis JS, Dulhunty JM, Cotta MO, Myburgh J, et al. Continuous versus intermittent betalactam infusion in severe sepsis: a meta-analysis of individual patient data from randomized trials. Am J Respir Crit Care Med. 2016;194(6):681-91.

19. Vardakas KZ, Voulgaris GL, Maliaros A, Samonis G, Falagas ME. Prolonged versus short-term intravenous infusion of antipseudomonal $\beta$-lactams for patients with sepsis: a systematic review and meta-analysis of randomised trials. Lancet Infect Dis. 2018;18(1):108-20.

20. Lee LS, Kinzig-Schippers M, Nafziger AN, Ma L, Sorgel F, Jones $\mathrm{RN}$, et al. Comparison of 30-min and 3-h infusion regimens for imipenem/cilastatin and for meropenem evaluated by Monte Carlo simulation. Diagn Microbiol Infect Dis. 2010;68(3):251-8.

21. Lee YR, Miller PD, Alzghari SK, Blanco DD, Hager JD, Kuntz KS. Continuous infusion versus intermittent bolus of beta-lactams in critically ill patients with respiratory infections: a systematic review and meta-analysis. Eur J Drug Metab Pharmacokinet. 2017;43:1-16.

22. Yang H, Zhang C, Zhou Q, Wang Y, Chen L. Clinical outcomes with alternative dosing strategies for piperacillin/tazobactam: a systematic review and meta-analysis. PLoS One. 2015;10(1):e0116769.

23. Cotta MO, Roberts JA, Lipman J. We need to optimize piperacillin-tazobactam dosing in critically ill patients-but how? Crit Care. 2016;20(1):163.

24. Roberts JA, Kumar A, Lipman J. Right dose, right now: customized drug dosing in the critically ill. Crit Care Med. 2017;45(2):331-6.

25. Roehr AC, Frey OR, Koeberer A, Fuchs T, Roberts JA, Brinkmann A. Anti-infective drugs during continuous hemodialysis-using the bench to learn what to do at the bedside. Int J Artif Organs. 2015;38(1):17-22.

26. Wong G, Briscoe S, Adnan S, McWhinney B, Ungerer J, Lipman J, et al. Protein binding of $\beta$-lactam antibiotics in critically ill patients: can we successfully predict unbound concentrations? Antimicrob Agents Chemother. 2013;57(12):6165-70.

27. Dellinger RP, Levy MM, Rhodes A, Annane D, Gerlach H, Opal SM, et al. Surviving Sepsis Campaign: international guidelines for management of severe sepsis and septic shock, 2012. Intensive Care Med. 2013;39(2):165-228.

28. Cockcroft DW, Gault H. Prediction of creatinine clearance from serum creatinine. Nephron. 1976;16(1):31-41.
29. Udy AA, Baptista JP, Lim NL, Joynt GM, Jarrett P, Wockner L, et al. Augmented renal clearance in the ICU: results of a multicenter observational study of renal function in critically ill patients with normal plasma creatinine concentrations. Crit Care Med. 2014;42(3):520-7.

30. Udy AA, Varghese JM, Altukroni M, Briscoe S, McWhinney BC, Ungerer JP, et al. Subtherapeutic initial beta-lactam concentrations in select critically ill patients: association between augmented renal clearance and low trough drug concentrations. Chest. 2012;142(1):30-9.

31. Udy AA, Lipman J, Jarrett P, Klein K, Wallis SC, Patel K, et al. Are standard doses of piperacillin sufficient for critically ill patients with augmented creatinine clearance? Crit Care. 2015;19(1):28.

32. Udy AA, Roberts JA, Lipman J. Implications of augmented renal clearance in critically ill patients. Nat Rev Nephrol. 2011;7(9):539.

33. Cho S-J, Yoon I-S, Kim D-D. Obesity-related physiological changes and their pharmacokinetic consequences. J Pharmaceutical Investig. 2013;43(3):161-9.

34. Yu Z, Pang X, Wu X, Shan C, Jiang S. Clinical outcomes of prolonged infusion (extended infusion or continuous infusion) versus intermittent bolus of meropenem in severe infection: a meta-analysis. PLoS One. 2018;13(7):e0201667.

35. Dulhunty JM, Roberts JA, Davis JS, Webb SA, Bellomo R, Gomersall $\mathrm{C}$, et al. Continuous infusion of beta-lactam antibiotics in severe sepsis: a multicenter double-blind, randomized controlled trial. Clin Infect Dis. 2012;56(2):236-44.

36. Dhaese SAM, Thooft ADJ, Farkas A, Lipman J, Verstraete AG, Stove V, et al. Early target attainment of continuous infusion piperacillin/tazobactam and meropenem in critically ill patients: a prospective observational study. J Crit Care. 2019;52:75-9.

37. Imani S, Buscher H, Marriott D, Gentili S, Sandaradura I. Too much of a good thing: a retrospective study of $\beta$-lactam concentration-toxicity relationships. J Antimicrob Chemother. 2017;72(10):2891-7.

38. Quinton MC, Bodeau S, Kontar L, Zerbib Y, Maizel J, Slama M, et al. Neurotoxic concentration of piperacillin during continuous infusion in critically ill patients. Antimicrob Agents Chemother. 2017;61(9):e00654-17.

39. Dulhunty JM, Roberts JA, Davis JS, Webb SA, Bellomo R, Gomersall $\mathrm{C}$, et al. A multicenter randomized trial of continuous versus intermittent $\beta$-lactam infusion in severe sepsis. Am J Respir Crit Care Med. 2015;192(11):1298-305.

40. Rutter WC, Cox JN, Martin CA, Burgess DR, Burgess DS. Nephrotoxicity during vancomycin therapy in combination with piperacillin-tazobactam or cefepime. Antimicrob Agents Chemother. 2017;61(2):e02089-16.

41. Hammond DA, Smith MN, Li C, Hayes SM, Lusardi K, Bookstaver PB. Systematic review and metaanalysis of acute kidney injury associated with concomitant vancomycin and piperacillin/ tazobactam. Clin Infect Dis. 2017;64(5):666-74.

42. Navalkele B, Pogue JM, Karino S, Nishan B, Salim M, Solanki $\mathrm{S}$, et al. Risk of acute kidney injury in patients on concomitant vancomycin and piperacillin-tazobactam compared to those on vancomycin and cefepime. Clin Infect Dis. 2016;64(2):116-23.

43. Roger C, Cotta MO, Muller L, Wallis SC, Lipman J, Lefrant JY, et al. Impact of renal replacement modalities on the clearance of piperacillin-tazobactam administered via continuous infusion in critically ill patients. Int J Antimicrob Agents. 2017;50(2):227-31.

44. Hoste EA, Bagshaw SM, Bellomo R, Cely CM, Colman R, Cruz $\mathrm{DN}$, et al. Epidemiology of acute kidney injury in critically ill patients: the multinational AKI-EPI study. Intensive Care Med. 2015;41(8):1411-23. 
45. Uchino S, Kellum JA, Bellomo R, Doig GS, Morimatsu H, Morgera S, et al. Acute renal failure in critically ill patients: a multinational, multicenter study. JAMA. 2005;294(7):813-8.

46. Choi G, Gomersall CD, Lipman J, Wong A, Joynt GM, Leung P, et al. The effect of adsorption, filter material and point of dilution on antibiotic elimination by haemofiltration: an in vitro study of levofloxacin. Int J Antimicrob Agents. 2004;24(5):468-72.

47. Tegeder I, Neumann F, Bremer F, Brune K, Lötsch J, Geisslinger G. Pharmacokinetics of meropenem in critically ill patients with acute renal failure undergoing continuous venovenous hemofiltration. Clin Pharmacol Ther. 1999;65(1):50-7.

48. Beumier M, Casu GS, Hites M, Seyler L, Cotton F, Vincent J-L, et al. $\beta$-lactam antibiotic concentrations during continuous renal replacement therapy. Crit Care. 2014;18(3):R105.

49. Roberts JA, Choi GY, Joynt GM, Paul SK, Deans R, Peake S, et al. SaMpling Antibiotics in Renal Replacement Therapy (SMARRT): an observational pharmacokinetic study in critically ill patients. BMC Infect Dis. 2016;16(1):103.

50. Pecly I, Genelhu V, Francischetti E. Renal functional reserve in obesity hypertension. Int J Clin Pract. 2006;60(10):1198-203.

51. Chagnac A, Weinstein T, Korzets A, Ramadan E, Hirsch J, Gafter U. Glomerular hemodynamics in severe obesity. Am J Physiol Renal Physiol. 2000;278(5):F817-22.

52. Alobaid AS, Brinkmann A, Frey OR, Roehr AC, Luque S, Grau $S$, et al. What is the effect of obesity on piperacillin and meropenem trough concentrations in critically ill patients? J Antimicrob Chemother. 2015;71(3):696-702.

53. Alobaid AS, Hites M, Lipman J, Taccone FS, Roberts JA. Effect of obesity on the pharmacokinetics of antimicrobials in critically ill patients: a structured review. Int J Antimicrob Agents. 2016;47(4):259-68.

54. Alobaid AS, Wallis SC, Jarrett P, Starr T, Stuart J, Lassig-Smith $\mathrm{M}$, et al. Effect of obesity on the population pharmacokinetics of meropenem in critically ill patients. Antimicrob Agents Chemother. 2016;60(8):4577-84.

55. Roberts JA, Lipman J. Optimal doripenem dosing simulations in critically ill nosocomial pneumonia patients with obesity, augmented renal clearance, and decreased bacterial susceptibility. Crit Care Med. 2013;41(2):489-95.

56. Hagel S, Fiedler S, Hohn A, Brinkmann A, Frey OR, Hoyer H, et al. Therapeutic drug monitoring-based dose optimisation of piperacillin/tazobactam to improve outcome in patients with sepsis (TARGET): a prospective, multi-centre, randomised controlled trial. Trials. 2019;20(1):330.

57. Lipman J, Brett SJ, De Waele JJ, Cotta MO, Davis JS, Finfer S, et al. A protocol for a phase 3 multicentre randomised controlled trial of continuous versus intermittent beta-lactam antibiotic infusion in critically ill patients with sepsis: BLING III. Crit Care Resusc. 2019;21(1):63-8.

58. Bush K, Macalintal C, Rasmussen B, Lee V, Yang Y. Kinetic interactions of tazobactam with beta-lactamases from all major structural classes. Antimicrob Agents Chemother. 1993;37(4):851-8.

59. Naicker S, Valero YCG, Meija JLO, Lipman J, Roberts JA, Wallis SC, et al. A UHPLC-MS/MS method for the simultaneous determination of piperacillin and tazobactam in plasma (total and unbound), urine and renal replacement therapy effluent. J Pharm Biomed Anal. 2018;148:324-33.

60. Chandorkar G, Huntington JA, Gotfried MH, Rodvold KA, Umeh O. Intrapulmonary penetration of ceftolozane/tazobactam and piperacillin/tazobactam in healthy adult subjects. J Antimicrob Chemother. 2012;67(10):2463-9.

61. Komuro M, Maeda T, Kakuo H, Matsushita H, Shimada J. Inhibition of the renal excretion of tazobactam by piperacillin. J Antimicrob Chemother. 1994;34(4):555-64.

\section{Affiliations}

\section{Daniel C. Richter ${ }^{1}$ (D) Otto Frey ${ }^{2} \cdot$ Anka Röhr $^{2}$ - Jason A. Roberts ${ }^{3,4,5,6}$. Andreas Köberer ${ }^{7}$. Thomas Fuchs ${ }^{7}$. Nikolaos Papadimas ${ }^{8}$. Monika Heinzel-Gutenbrunner ${ }^{9}$. Thorsten Brenner ${ }^{1}$. Christoph Lichtenstern ${ }^{1}$. Markus A. Weigand ${ }^{1} \cdot$ Alexander Brinkmann ${ }^{7}$}

1 Department of Anesthesiology and Critical Care, Heidelberg University Hospital, Im Neuenheimer Feld 110, 69120 Heidelberg, Germany

2 Department of Clinical Pharmacy, Heidenheim Hospital, Schloßhaustraße 100, 89522 Heidenheim, Germany

3 University of Queensland Centre for Clinical Research, The University of Queensland, St Lucia, Brisbane, QLD 4072, Australia

4 Centre for Translational Anti-infective Pharmacodynamics, School of Pharmacy, The University of Queensland, 20 Cornwall St, Woolloongabba, Brisbane, QLD 4102, Australia

5 Royal Brisbane and Women's Hospital, Brisbane, Australia
6 Royal Brisbane and Women's Hospital, Herston 4029, QLD, Australia

7 Department of Anesthesiology, Heidenheim Hospital, Schloßhaustraße 100, 89522 Heidenheim, Germany

8 Department of Urology, Heidenheim Hospital, Schloßhaustraße 100, 89522 Heidenheim, Germany

9 MH-Statistical Consulting, Bienenweg 8, 35041 Marburg, Germany 